\title{
Hearing in the starling (Sturnus vulgaris): Absolute thresholds and critical ratios
}

\author{
ROBERT J. DOOLING, KAZUO OKANOYA, and JANE DOWNING \\ University of Maryland, College Park, Maryland \\ and \\ STEWART HULSE \\ Johns Hopkins University, Baltimore, Maryland
}

\begin{abstract}
Operant conditioning and a psychophysical tracking procedure were used to measure auditory thresholds for pure tones in quiet and in noise for a European starling. The audibility curve for the starling is similar to the auditory sensitivity reported earlier for this species using a heartrate conditioning procedure. Masked auditory thresholds for the starling were measured at a number of test frequencies throughout the bird's hearing range. Critical ratios (signal-to-noise ratio at masked threshold) were calculated from these pure tone thresholds. Critical ratios increase throughout the starling's hearing range at a rate of about $3 \mathrm{~dB}$ per octave. This pattern is similar to that observed for most other vertebrates. These results suggest that the starling shares a common mechanism of spectral analysis with many other vertebrates, including the human.
\end{abstract}

Bird vocalizations are some of the most complex acoustic signals known to man. Partly for this reason, the European starling is becoming a favorite subject for both behavioral and physiological investigations of the auditory processing of complex sounds (Hulse \& Cynx, 1984a, 1984b; Leppelsack, 1978). Less is known about the basic auditory sensitivity of this species, but there are two reports in the literature of absolute thresholds in the starling. One report relied on a classical conditioning procedure (Trainer, 1946) and the other used an indirect procedure involving conditioned heart-rate changes (Kuhn, Muller, Leppelsack, \& Schwartzkopff, 1982). The same heart-rate conditioning procedure has also been used to measure frequency difference thresholds in the starling (Kuhn, Leppelsack, \& Schwartzkopff, 1980).

Inasmuch as the starling is becoming a popular subject for acoustic experiments, it is important that auditory thresholds be examined using a more direct procedure. The purpose of the present experiment was to measure the auditory sensitivity of the starling in quiet and in noise, using operant conditioning and an automated psychophysical tracking procedure. Thresholds in quiet would confirm the accuracy of previous studies of absolute thresholds in these species. Thresholds in noise would have relevance for broader questions about the perception of complex sounds (Scharf, 1970) and whether spectral resolving power in the starling is similar to that typically found for other vertebrates.

We thank S. Brown and T. Park for helpful criticisms. This work was supported by an NSF Grant to R. Dooling. Address correspondence to R. Dooling, Psychology Department, University of Maryland, College Park, MD 20742.

\section{METHOD}

Subject

The bird used in this experiment was a male starling obtained from the laboratory of Stewart Hulse of Johns Hopkins University. This bird had been used in previous experiments on complex sound perception before being trained for the hearing tests described below. During the present experiment, the starling was housed in the aviaries at the University of Maryland and kept on a normal day/night cycle correlated with the season.

\section{Apparatus}

The apparatus has been described previously in detail (Okanoya \& Dooling, 1985). A custom-made operant chamber with a food hopper and two response keys constructed of microswitches and LEDs were used. A standard pigeon grain hopper delivered food. The test cage was suspended in a large anechoic chamber measuring $5 \times 2.5 \times 2.5 \mathrm{~m}$. Acoustic stimuli were delivered by Coulbourn Instrument modules and the conduct of the experiment was under the control of a microcomputer.

Stimulus calibration was performed using a General Radio Model 1982 sound-level meter with octave band filters. Tone intensity was measured by placing the microphone in the position normally occupied by the bird's head during testing. Ambient noise level was measured in the same way. In addition, a B\&K sound-level meter with $1 / 3$ octave filters was used to obtain a more precise estimate of the spectrum level of white noise. A spectrum analyzer ensured that the distribution of energy within each $1 / 3$ octave band was relatively flat.

\section{Training and Testing Procedure}

The training and testing procedure has also been described in detail previously (Okanoya \& Dooling, 1985). Briefly, the starling was trained to peck at two small microswitch keys in order to receive food reinforcement. A peck on one key resulted in the presentation of a tone with a probability of .10 . Detection of the tone was reported by a peck on a second key. Correct responses resulted in a lowering of tone sound pressure level (SPL) for the next trial, whereas failure to respond resulted in the raising of tone SPL for the next trial. The bird was deprived of food for a few hours before each daily session. When the bird attained a level of $90 \%$ correct combined with a false alarm rate of less than $10 \%$ over a session of 40 trials, actual testing began. 
Threshold Determination

Thresholds were determined with a tracking procedure. For the first 10 trials of the tracking session, tone intensity was fixed at a level 10-50 dB above the bird's suspected threshold. For the next 30 trials, the stimulus level was contingent on the bird's performance. Threshold was estimated from the tracking reversal points that occurred during the last 10 trials of a daily session by taking the average of the last four reversals that occurred during the last 10 trials. The final threshold estimate at any given frequency was based on threshold estimates from a number of sessions.

Once thresholds for pure tones were obtained in quiet, a background masker of white noise was added and critical ratios (signal-to-noise ratio at masked threshold) were obtained at six test frequencies between $500 \mathrm{~Hz}$ and $5.7 \mathrm{kHz}$. The spectrum level of white noise used as a masker was well above the bird's absolute threshold at all test frequencies.

\section{RESULTS}

The behavioral audibility curve for our starling is shown in Figure 1. The thresholds obtained with our operant procedure were slightly higher at low frequencies and slightly lower at high frequencies than the pure tone thresholds reported for the starling using the heart-rate conditioning procedure (Kuhn et al., 1982). Also shown in Figure 1 are thresholds for the starling obtained using a classical conditioning procedure (Trainer, 1946). Except at very low frequencies, the thresholds for our starling are in reasonably good agreement with those obtained with the classical conditioning procedure.

Critical ratios for the starling are shown in Figure 2. The signal-to-noise ratio at masked threshold increases at the rate of $3 \mathrm{~dB}$ per octave, as is the case for many other vertebrates, including the human (Dooling, 1980, 1982). For comparison, data for the human are also shown (Hawkins \& Stevens, 1950). Also shown is a critical ratio function for the starling estimated from frequency difference limen data obtained with a heart-rate condition-

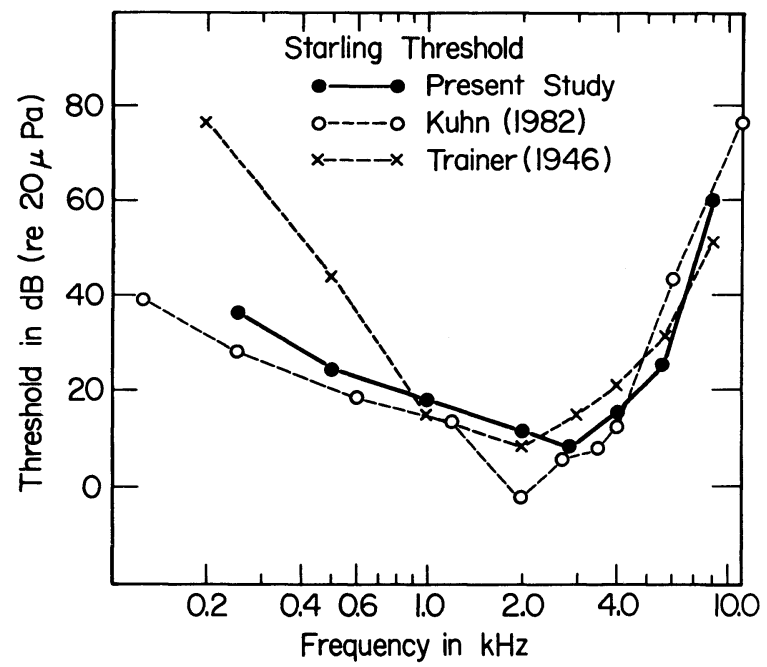

Figure 1. Audibility curve of the starling using an operant conditioning and tracking procedure is compared to pure tone thresholds obtained using conditioned heart-rate (replotted from Kuhn et al., 1982) and classical conditioning (replotted from Trainer, 1946).

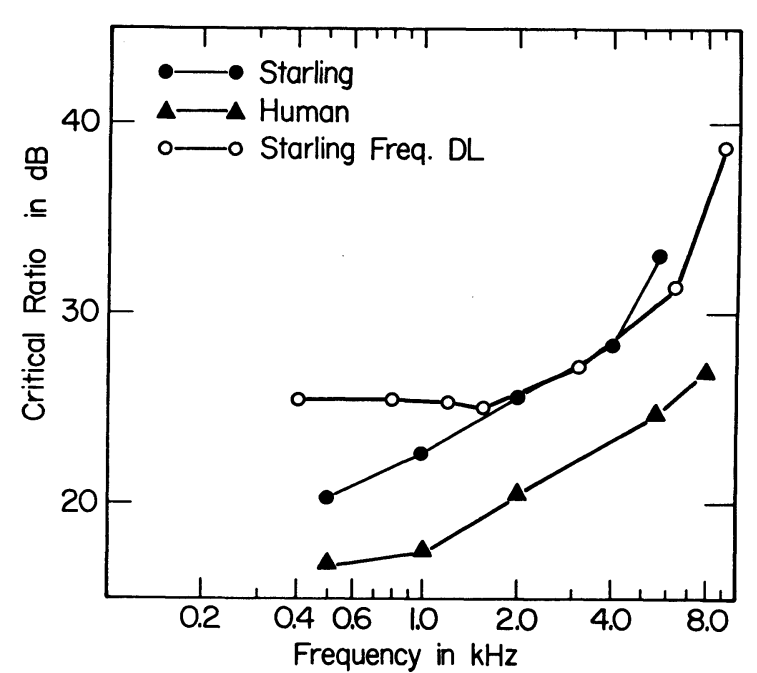

Figure 2. Critical ratio data for the starling obtained with the present procedure are compared to a hypothetical critical ratio function generated from frequency difference limen data of Kuhn et al. (1980) according to the formula: critical ratio $=10 \log (40 \times d f)$. For comparison, critical ratio data for the human are also shown (replotted from Hawkins \& Stevens, 1950).

ing procedure (Kuhn et al., 1980). As is the case with most vertebrates, critical ratios parallel the frequency difference limen function, at least over the middle of the starling's hearing range. This correspondence is strongly suggestive of a common mechanism underlying frequency resolution and the detection of signals in noise.

\section{DISCUSSION}

These findings are significant for several reasons. First, hearing thresholds obtained with operant conditioning in combination with an automated tracking procedure confirm those obtained using heart-rate and classical conditioning. Second, the starling is like most other vertebrates in the slope of its critical ratio function, with critical ratios increasing at the rate of $3 \mathrm{~dB}$ per octave over most of the hearing range. Third, electrophysiological data from auditory fibers in the avian auditory periphery often highlight the similarities in stimulus coding between mammals and birds (Sachs, Woolf, \& Sinnott, 1980), and the present data support these similarities.

Interestingly, the starling is distinctly different from the only other bird that has been tested for critical ratios-the budgerigar (Melopsittacus undulatus) (Dooling \& Saunders, 1975). The reason for this difference between the starling and the budgerigar is not yet clear.

The present results have particular relevance for understanding the perception of complex pitch patterns by starlings. The relation between test frequency and critical ratios in the region of $1.5-4.0 \mathrm{kHz}$ indicates that the starling auditory system encodes frequency change in a logarithmic fashion. In most vertebrates, the foundation for this frequency encoding scheme begins in the auditory periphery with the spatial arrangement of frequency along the basilar membrane. There is also anatomical evidence for a logarithmic organization of frequency along the avian basilar papilla (Dooling, 1980; Saito, 1980).

Recent studies have shown that starlings perceive complex serial pitch patterns differently than do humans (Hulse, Cynx, \& Humpal, 1984a, 1984b). Working with tone sequences, Hulse and his colleagues showed that while starlings could learn to make relative pitch discriminations, they exhibited an overwhelming bias for relying on absolute frequency or pitch cues in solving a discrimination problem. These results are con- 
trary to results from pitch transformation experiments in humans. In view of the present findings, it is likely that more central or integrative processes, rather than simple frequency encoding by the peripheral auditory system, are involved in these species differences.

\section{REFERENCES}

Dooling, R. (1980). Behavior and psychophysics of hearing in birds. In A. Popper \& R. Fay (Eds.), Comparative studies of hearing in vertebrates (pp. 261-288). New York: Springer-Verlag.

Dooling, R. (1982). Auditory perception in birds. In D. Kroodsma \& E. Miller (Eds.), Acoustic communication in birds (Vol. 1, pp. 95130). New York: Academic Press.

Dooling, R., \& SAUNDERS, J. (1975). Hearing in the parakeet (Melopsittacus undulatus): Absolute thresholds, critical ratios, frequency difference limens, and vocalizations. Journal of Comparative \& Physiological Psychology, 88, 1-20.

Hawkins, J. E., \& Stevens, S. S. (1950). The masking of pure tones and speech by white noise. Journal of the Acoustical Society of America, 22, 6-13.

Hulse, S. H., CynX, J., \& Humpal, J. (1984a). Absolute and relative pitch discrimination in serial pitch perception by birds. Journal of $E x$ perimental Psychology: General, 113, 38-54.

Hulse, S. H., CYNX, J., \& Humpal, J. (1984b). Cognitive processing of pitch and rhythm structures by birds. In H. Roitblat, T. Bever, \& H. Terrace (Eds.), Animal cognition (pp. 183-198). Hillsdale, NJ: Erlbaum.
Kuhn, A., Leppelsack, H.-J., \& SchwartzkopfF, J. (1980). Measurement of frequency discrimination in the starling (Sturnus vulgaris) by conditioning of heart rate. Naturwissenschaften, 67, 102-103.

Kuhn, A., Muller, C. M., Leppelsack, H.-J., \& SCHWARTZKopfF, J. (1982). Heart-rate conditioning used for determination of auditory thresholds in the starling. Naturwissenschaften, 69, 245-246.

LEPPELSACK, H.-J. (1978). Unit responses to species-specific sounds in the auditory forebrain centers of birds. Federation Proceedings, 37, 2336-2341.

OKanoya, K., \& Dooling, R. (1985). Colony differences in auditory thresholds in the canary (Serinus canarius). Journal of the Acoustical Society of America, 78, 1170-1176.

Sachs, M. B., Woolf, N. K., \& Sinnotr, J. M. (1980). Response properties of neurons in the avian auditory system: Comparisons with mammalian homologues and consideration of the neural encoding of complex stimuli. In A. Popper \& R. Fay (Eds.), Comparative studies of hearing in vertebrates (pp. 323-353). New York: Springer-Verlag.

SaITo, N. (1980). Structure and function of the avian ear. In A. Popper \& R. Fay (Eds.), Comparative studies of hearing in vertebrates (pp. 241-260). New York: Springer-Verlag.

Scharf, B. (1970). Critical bands. In J. V. Tobias (Ed.), Foundations of modern auditory theory (Vol. 1, pp. 159-202). New York: Academic Press.

Trainer, J. E. (1946). The auditory acuity of certain birds. Unpublished doctoral dissertation. Cornell University, Ithaca, NY.

(Manuscript received for publication June 26, 1986). 